\title{
Three-Dimensional Neuronal Structure of Human Cerebral Cortex Determined by Synchrotron-Radiation Microtomography
}

\author{
Rino Saiga ${ }^{1}$, Susumu Takekoshi ${ }^{2}$, Chie Inomoto $^{2}$, Naoya Nakamura ${ }^{2}$, Akio Tsuboi $^{2}$, Motoki Osawa $^{2}$, \\ Makoto Arai ${ }^{3}$, Kenichi Oshima ${ }^{3}$, Masanari Itokawa ${ }^{3}$, Kentaro Uesugi ${ }^{4}$, Akihisa Takeuchi ${ }^{4}$, Yasuko \\ Terada $^{4}$, Yoshio Suzuki ${ }^{4}$ and Ryuta Mizutani ${ }^{1}$ \\ 1. Department of Applied Biochemistry, Tokai University, Hiratsuka, Kanagawa 259-1292, Japan. \\ 2. Tokai University School of Medicine, Isehara, Kanagawa 259-1193, Japan. \\ 3. Tokyo Metropolitan Institute of Medical Science, Setagaya, Tokyo 156-8506, Japan. \\ 4. Japan Synchrotron Radiation Research Institute (JASRI/SPring-8), Sayo, Hyogo 679-5198, Japan.
}

Neuronal circuits are responsible for brain functions including cognition, reasoning, and decision making. Neurons constitute neuronal circuits by forming three-dimensional networks in the cerebral cortex tissue. Therefore, the functional mechanism of human brain can be revealed by visualizing and analyzing the three-dimensional structure of the cerebral cortex. Here, we report on the threedimensional structure of human cerebral-cortex tissue, as determined by synchrotron-radiation microtomography at resolutions up to $100 \mathrm{~nm}$.

Post-mortem cerebral tissues were collected with informed consent from the legal next of kin using protocols approved by ethical committees of the related organizations. The cerebral tissues were subjected to Golgi impregnation, as described previously [1]. The stained tissues were sequentially immersed in ethanol, $n$-butylglycidyl ether, and epoxy resin. The samples were transferred to a borosilicate glass capillary filled with resin and kept at $90^{\circ} \mathrm{C}$ for $40 \mathrm{~h}$ to cure the resin.

X-ray microtomography equipped with Fresnel zone plate (FZP) optics was performed at the BL37XU beamline of the SPring-8 synchrotron radiation facility. An FZP with an outermost zone width of $50 \mathrm{~nm}$ and diameter of $250 \mu \mathrm{m}$ [2] was used as an objective lens, and an x-ray guide tube [3] as a beam condenser. A total of 900 transmission images were recorded with a CMOS-based detector using monochromatic radiation of $8 \mathrm{keV}$. Tomographic sections were reconstructed with the convolution back-projection method of the RecView program (available from http://www.el.u-tokai.ac.jp/ryuta/). Spatial resolution was estimated to be $100 \mathrm{~nm}$ by using test objects [4].

The obtained three-dimensional structure of human cerebral tissue is shown in Figure 1. Neurons and neuronal processes were clearly visualized as a three-dimensional distribution of x-ray attenuation coefficients. Dendritic spines were observed as small protruding structures from the dendrites. These spines have claviform heads with longitudinal lengths of 300-950 nm and cross-sectional diameters of 150-350 nm. The heads are connected to the dendrites through thin cords with typical widths of $150 \mathrm{~nm}$ and lengths of 300-1500 nm. These spines are responsible for neurotransmission in neural circuits.

Simple-projection x-ray microtomography with a wider field of view $(1 \mathrm{~mm})$ was performed at the BL20XU beamline of SPring-8. A total of 1800 transmission images per dataset were recorded using 12 $\mathrm{keV}$ radiation. The spatial resolution was estimated to be $1.2 \mu \mathrm{m}$ by using test objects. The obtained structure (Fig. 2a) illustrated complicated neuronal networks that cannot be comprehended at a glance. As a result, the image had to be further analyzed to reveal the neuronal circuits embedded in the cerebral tissue. Such neuronal network models can be built by placing and connecting nodes in the three- 
dimensional map of x-ray attenuation coefficients that represent electron densities. Series of nodes corresponding to neuronal processes and somas were traced to construct skeletonized models of neurons (Fig. 2b). Since the resultant models are represented in three-dimensional Cartesian coordinates, the distances between neuronal processes or somas can be readily calculated from the coordinates. This will allow us to determine neuronal circuits in the human brain tissue by analyzing the positional relationships of the neurons $[1,5]$.

[1] R Mizutani et al, Cerebral Cortex 20 (2010), 1739-1748.

[2] Y Suzuki et al, Jpn. J. Appl. Phys. 44 (2005), 1994-1998.

[3] Y Suzuki et al, J. Phys. Conf. Ser. 463 (2013), 012028.

[4] R Mizutani et al, Nucl. Instrum. Meth. A 621 (2010), 615-619.

[5] This work was supported in part by Grants-in-Aid for Scientific Research from the Japan Society for the Promotion of Science (nos. 21611009, 25282250, and 25610126). The synchrotron radiation experiments were performed at SPring-8 with the approval of the Japan Synchrotron Radiation Research Institute (JASRI) (proposal nos. 2013A1384, 2014A1057, and 2014B1083).

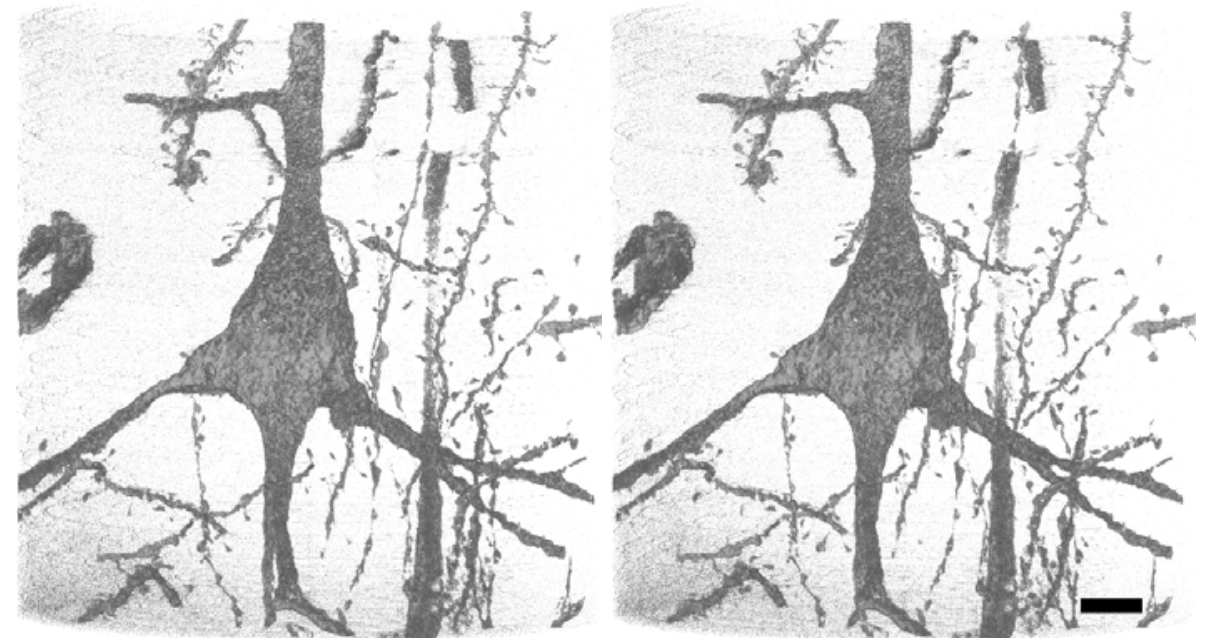

Figure 1. Stereo rendering of the three-dimensional structure of a pyramidal neuron and neuronal processes in the human cerebral cortex. Scale bar: $5.0 \mu \mathrm{m}$.

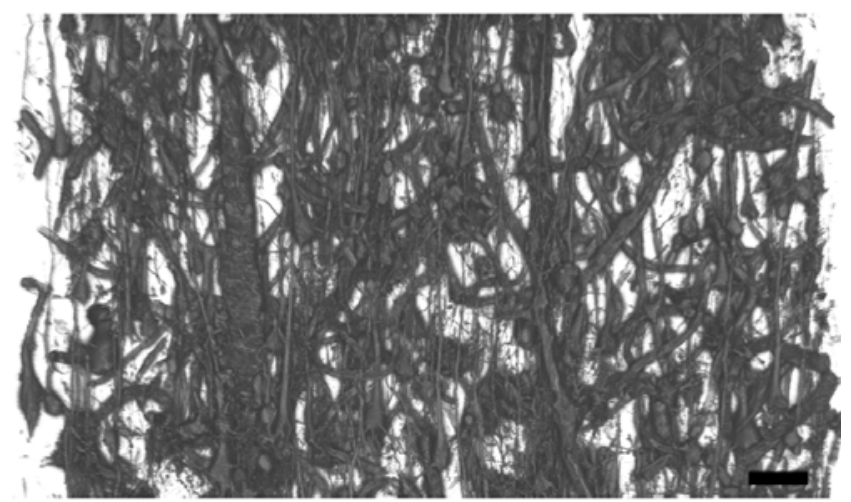

(a)

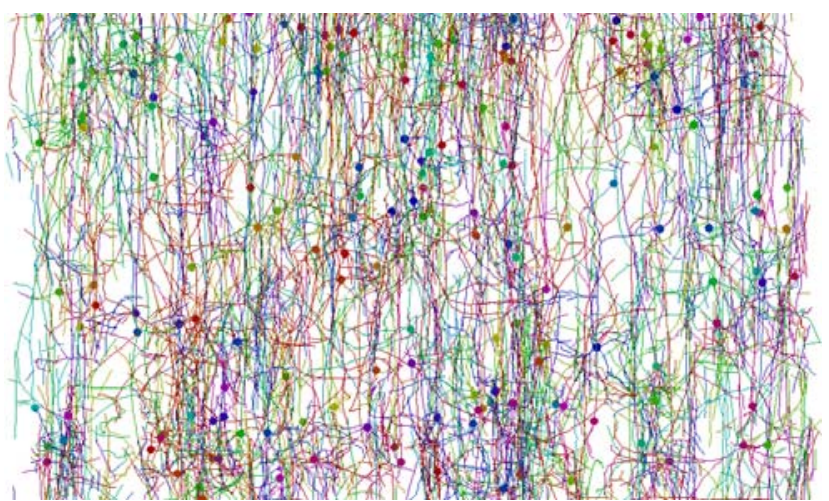

(b)

Figure 2. (a) Three-dimensional structure of human cerebral tissue. Scale bar: $50 \mu \mathrm{m}$. (b) Skeletonized model of the tissue structure. Neurons are color-coded. Soma positions are indicated with closed circles. 